\title{
THE CONVEXITY OF A DOMAIN AND THE SUPERHARMONICITY OF THE SIGNED DISTANCE FUNCTION
}

\author{
D. H. ARMITAGE AND Ü. KURAN
}

\begin{abstract}
Let $D$ be a domain in $\mathbf{R}^{N}$ with nonempty boundary $\partial D$ and let $u$ be the signed distance function from $\partial D$, i.e. $u= \pm$ dist according as we are in or outside $\bar{D}$. We prove that, for any $N \geqslant 2, u$ is superharmonic in $\mathbf{R}^{N}$ if and only if $D$ is convex. When $N=2$, this criterion requires the superharmonicity of $u$ in $D$ only.
\end{abstract}

1. Throughout this paper $D$ will denote a proper subdomain of the Euclidean space $\mathbf{R}^{N}$, where $N \geqslant 2$. Thus the boundary $\partial D$ of $D$ in $\mathbf{R}^{N}$ is not empty and we can define the distance function $d$ from $\partial D$. The signed distance function $u$ in $\mathbf{R}^{N}$ is defined by

$$
u= \begin{cases}d & \text { in } \bar{D} \\ -d & \text { in } D^{\prime}\end{cases}
$$

where $\bar{D}$ is the closure of $D$ in $\mathbf{R}^{N}$ and $D^{\prime}=\mathbf{R}^{N} \backslash \bar{D}$.

Our main result is the following

THEOREM 1. The function $u$ is superharmonic in $\mathbf{R}^{N}$ if and only if $D$ is convex.

The "if" part of Theorem 1 must be known, at least tacitly; cf. Fuchs [1, p. 11]. For completeness we sketch a proof. For every support hyperplane $H$ of $\bar{D}$ let $u_{H}$ be the signed distance function from $H$ such that $u_{H}>0$ in $D$ and $u_{H}$ is harmonic in $\mathbf{R}^{N}$. Then $u=\inf _{H} u_{H}$ and it follows that $u$ is superharmonic (in fact, $u$ is concave) in $\mathbf{R}^{N}$, since the $u_{H}$ are all harmonic and $u \in \mathscr{C}\left(\mathbf{R}^{N}\right)$. The proof of the "only if" part of Theorem 1 (cf. §3) is more involved and requires two preliminary lemmas $(\S 2)$.

We note that, for example, if $D$ is the punctured ball $D=\left\{X \in \mathbf{R}^{N}: 0<r=\|X\|\right.$ $<1$, then $u$ is superharmonic in $D^{\prime}$ but not in $D$. With this motivation we now state

THEOREM 2. If $D$ is a planar domain and $d$ is superharmonic in $D$, then $D$ is convex. In higher dimensions, neither $D$ nor $\bar{D}$ need be convex.

THEOREM 3. Let $F$ be a proper closed subset of $\mathbf{R}^{N}$, where $N \geqslant 2$, and let $d$ be the distance from $\partial F$. Then $d$ is subharmonic in $F^{\prime}$ if and only if $F$ is convex.

The $N=2$ and $N \geqslant 3$ cases of Theorem 2 are proved in $\S \S 4$ and 5, Theorem 3 in $\S 3$.

Received by the editors July 7, 1983.

1980 Mathematics Subject Classification. Primary 31B05; Secondary 52A20.

Kev words and phrases. Superharmonic function, distance, convex set.

(C)1985 American Mathematical Society $0002-9939 / 85 \$ 1.00+\$ .25$ per page 
2. Lemma 1. Let $D \subset \mathbf{R}^{N}$ be such that $D \neq \operatorname{int}(\bar{D})$. Then $u$ is not superharmonic in $\mathbf{R}^{N}$.

We denote the mean-value of $u$ on $S\left(X_{0}, r\right)=\left\{X:\left\|X-X_{0}\right\|=r\right\}$ by $M\left(u, X_{0}, r\right)$.

To prove Lemma 1 , choose $X_{0} \in \partial D$ and $r_{0}>0$ so that $B\left(X_{0}, r_{0}\right)=\{X: \| X-$ $\left.X_{0} \|<r_{0}\right\} \subset \operatorname{int}(\bar{D}) \subset \bar{D}$. Clearly $M\left(u, X_{0}, r\right)>0$ if $0<r<r_{0}$; thus if $u$ were superharmonic we must have $u\left(X_{0}\right)>0$.

Lemma 2. Let $Y_{1}, Y_{2}$ be distinct points in $\mathbf{R}^{N}$ such that $\left\|Y_{1}\right\|=\left\|Y_{2}\right\|$. Let $r_{1}, r_{2}$ denote the distances of a point from $Y_{1}, Y_{2}$, respectively, and define $v$ in $\mathbf{R}^{N}$ by $v=r_{1} \wedge r_{2}$. Then there exists a positive number $r_{0}$ such that $v(O)>M(v, O, r)$ for all $r$ in $\left(0, r_{0}\right)$.

By using a magnification, we may suppose that $\left\|Y_{1}\right\|=\left\|Y_{2}\right\|=1$, and by rotating the axes, we may suppose further that $Y_{1}=(\cos \phi, \sin \phi, 0, \ldots, 0)$ and $Y_{2}=$ $(-\cos \phi, \sin \phi, 0, \ldots, 0)$, where $0 \leqslant \phi<\pi / 2$.

If $X=\left(x_{1}, \ldots, x_{N}\right) \in \mathbf{R}^{N}$ and $r=\|X\|$, then, writing

$$
f(X)=r^{2}-2\left|x_{1}\right| \cos \phi-2 x_{2} \sin \phi
$$

we have

Hence

$$
v(X)=(1+f(X))^{1 / 2} \leqslant 1+\frac{1}{2} f(X) .
$$

$$
M(v, O, r) \leqslant 1+\frac{1}{2} M(f, O, r)=1+\frac{1}{2} r^{2}-(\cos \phi) M\left(\left|x_{1}\right|, O, r\right)
$$

Since $M\left(\left|x_{1}\right|, O, r\right)$ is a positive multiple of $r$ and $\cos \phi>0$, we have $M(v, O, r)<1$ $=v(O)$ when $r$ is small.

3. To prove the "only if" in Theorem 1 , suppose that $D$ is not convex. If $\bar{D}$ is convex, then Lemma 1 implies that $u$ is not superharmonic in $\mathbf{R}^{N}$, since then $\operatorname{int}(\bar{D})$ is convex [2, Theorem 1.11] and so $D \neq \operatorname{int}(\bar{D})$.

Now suppose that $\bar{D}$ is nonconvex. A key result for this case is Motzkin's theorem, which states that a proper closed subset $F$ of $\mathbf{R}^{N}$ is convex if and only if each point of $\mathbf{R}^{N}$ has a unique nearest point of $F$ (cf. [2, Theorem 7.8]). Hence, taking $F=\bar{D}$, we may assume that (by translating the origin, if necessary) $O \in D^{\prime}$ and that there exist distinct points $Y_{1}, Y_{2}$ of $\bar{D}$ such that $d(O)=\left\|Y_{1}\right\|=\left\|Y_{2}\right\|>0$. Define $v$ in $\mathbf{R}^{N}$ by $v(X)=\left\|X-Y_{1}\right\| \wedge\left\|X-Y_{2}\right\|$. By Lemma 2 , there exists $r_{0}>0$ such that $v(O)>M(v, O, r)$ whenever $0<r<r_{0}$. Also, $\overline{B(O, r)} \subset D^{\prime}$ for one of these $r$. Since $v(X) \geqslant d(X)$ for all $X$ in $D^{\prime}$ with equality when $X=O$, we obtain

$$
u(O)=-d(O)=-v(O)<-M(v, O, r) \leqslant-M(d, O, r)=M(u, O, r),
$$

so that $u$ is not superharmonic in $D^{\prime}$.

The argument in the last paragraph (with $\bar{D}$ replaced by $F$ ) proves the "only if" in Theorem 3. The proof of "if" in Theorem 3 is similar to the proof of "if" in Theorem $1(\$ 1)$.

4. To prove the plane case $(N=2)$ of Theorem 2, we suppose that $D$ is nonconvex in $\mathbf{R}^{2}$ and show that $d$ is not superharmonic in $D$. There exist a point $Y_{0}$ of $\partial D$, a positive number $\varepsilon$ and a closed half-plane $P$ with $Y_{0}$ on $\partial P$ such that

$$
\left.P \cap \overline{\left(B\left(Y_{0}, \varepsilon\right)\right.} \backslash\left\{Y_{0}\right\}\right) \subset D
$$


cf. [2, Theorem 4.8]. Without loss of generality, suppose that $Y_{0}=O$ and $P=\{X$ : $\left.x_{2} \geqslant 0\right\}$. Let $X_{0}=(0, \varepsilon / 4)$ and $B=B\left(X_{0}, \varepsilon / 8\right)$. If $X=\left(x_{1}, x_{2}\right) \in B$ and $x_{1} \neq 0$, then $d(X)>x_{2}$. Hence, by the area mean-value equality for the function $x_{2}$,

$$
\int_{B} d(X) d X>\int_{B} x_{2} d X=\pi(\varepsilon / 8)^{2}(\varepsilon / 4)=\pi(\varepsilon / 8)^{2} d\left(X_{0}\right),
$$

so that the area mean-value inequality for the superharmonicity of $d$ fails at $X_{0}$.

5. Here we show by an example that in higher dimensions $(N \geqslant 3)$ the superharmonicity of $u$ in $D$ does not necessarily imply the convexity of $D$, nor even of $\bar{D}$.

Let $\Omega$ denote the torus in $\mathbf{R}^{3}$ obtained by rotating the disc $\omega=\left\{\left(0, x_{2}, x_{3}\right)\right.$ : $\left.\left(x_{2}-a\right)^{2}+x_{3}^{2}<1\right\}$, where $a \geqslant 2$, about the $x_{3}$-axis. In the case $N=3$ let $D=\Omega$, and in the case $N \geqslant 4$ let $D=\Omega \times \mathbf{R}^{N-3}$. Clearly $D$ is not convex, and neither is $\bar{D}$. We shall show, however, that $d$ is superharmonic in $D$.

With a point $X$ (in $\mathbf{R}^{N}$ ) we associate plane polar coordinates $(r, \theta)$ such that $x_{1}=r \cos \theta$ and $x_{2}=r \sin \theta$ and we put $\rho=\rho(X)=\left(x_{3}^{2}+(r-a)^{2}\right)^{1 / 2}$. Then $D=\{X: \rho<1\}$ and $\partial D=\{X: \rho=1\}$.

If $X \in D$, then, in finding $d(X)$, we may suppose that $\left(x_{1}, x_{2}, x_{3}\right) \in \omega$. Let $X_{0}=(0, a, 0, \ldots, 0)$. Then $B\left(X, 1-\left\|X-X_{0}\right\|\right) \subset B\left(X_{0}, 1\right) \subset D$ and so $d(X) \geqslant 1-$ $\left\|X-X_{0}\right\|=1-\rho$. If $X=X_{0}$, then clearly $d(X)=1$; if $X \neq X_{0}$, then the point $Y_{0}$ such that $\left\|Y_{0}-X_{0}\right\|=1$ and $X_{0}, X, Y_{0}$ are collinear (in that order) belongs to $\partial D$, so that $d(X) \leqslant\left\|X-Y_{0}\right\|=1-\rho$. Hence, in all cases, $d(X)=1-\rho$.

Let $G=\{X: \rho=0\}$. We show first that $d$ is superharmonic in $D \backslash G$ by computing the Laplacian

$$
\Delta d(X)=-\Delta \rho=-\left\{\frac{\partial^{2} \rho}{\partial x_{3-}^{2}}+r^{-1} \frac{\partial \rho}{\partial r}+\frac{\partial^{2} \rho}{\partial r^{2}}\right\}=\frac{a-2 r}{r \rho} ;
$$

as we have $2 r>2(a-1) \geqslant a$, we get $\Delta d<0$. Hence $d$ is superharmonic in $D \backslash G$ and therefore satisfies the weak mean-value inequality in $D \backslash G$ (that is, if $S(X, r) \subset$ $D \backslash G$, then $d(X) \geqslant M(d, X, r))$. Further, $d$ takes its maximum value at each point of $G$ and therefore the mean-value inequality holds on $G$, too. As $d$ is continuous, it follows that $d$ is superharmonic in $D$.

\section{REFERENCES}

1. W. H. J. Fuchs, Topics in the theory of functions of one complex variable, Van Nostrand, Princeton, N. J., 1967.

2. F. A. Valentine, Convex sets, McGraw-Hill, New York, 1964.

Department of Pure Mathematics, The Queen's University, Belfast, Northern Ireland

Department of Pure Mathematics, The University, Liverpool, England 